\title{
Penile fracture: Retrospective analysis of our case history with long-term assessment of the erectile and sexological outcome
}

\author{
Nicola Pavan ${ }^{1}$, Giorgia Tezzot ${ }^{1}$, Giovanni Liguori ${ }^{1}$, Renata Napoli ${ }^{1}$, Paolo Umari ${ }^{1}$, Michele Rizzo ${ }^{1}$, \\ Giovanni Chiriacò $^{1}$, Gaetano Chiapparrone ${ }^{1}$, Francesca Vedovo ${ }^{1}$, Michele Bertolotto ${ }^{2}$, Carlo Trombetta ${ }^{1}$ \\ ${ }^{1}$ Urology Department, Cattinara Hospital, University of Trieste, Italy; \\ ${ }^{2}$ Radiology Department, Cattinara Hospital, University of Trieste.
}

\begin{abstract}
Summary Objectives: To review the cases of patients with suspected penile fracture and asses erectile and sexological outcomes. Materials and methods: from 1987 to 2013 presented to the Urology Clinic of Trieste and at the AIED of Pordenone a total of 41 cases that were divided into two groups according to the timing of treatment: 18 patients with anamnestic diagnosis of penile fracture treated nonimmediately and 23 patients treated immediately after the trauma. For all patients we evaluated the type of treatment adopted, the occurrence of complications and reoperations and the follow-up. The erectile function was also evaluated through the IIEF, as well as the psychological impact of the trauma on social and sex life, using a psycho-sexological questionnaire.

Results: Among patients treated immediately after the trauma 14 were subjected to surgery. About a year after surgery, penile curvature was reported in 1 patient, pain in 3 patients, urinary disorders in 1 patient, while none reported erectile dysfunction (ED). Out of these, only 3 patients underwent reoperation. Among those treated conservatively 1 patient reported curvature, 1 patient reported pain and none reported ED. Among patients who were admitted at a later date, 14 reported curvature and 4 reported pain whereas urinary disorders were reported in 1 and ED in 4 patients. From a psychological point of view, the trauma caused in most cases a fear of new trauma and of repercussions on erectile function and sensitivity. Conclusions: The diagnosis is mainly clinical; however, radiological investigation is essential to confirm the diagnosis, assess the site and extent of the trauma and possible urethral involvement, so as to plan the most appropriate treatment. In addition, immediate treatment leads to better long-term results, with a lower incidence of ED and penile curvature. Psychologically, penile trauma intensifies the fear of reoccurrence; it decreases, however, with the passage of time.
\end{abstract}

KEY WORDS: Penile fracture; Outcomes; Penile surgery; Penile ultrasound; Erectile deficiency.

Submitted 4 September 2014; Accepted 31 October 2014

No conflict of interest declared.

\section{INTRODUCTION}

The penile fracture is a more or less extensive lesion of the tunica albuginea and the underlying corpora cavernosa. Usually the lesion involves only a cavernous body, but in some cases both can be damaged $(1,2)$. In $20-30 \%$ of cases, the tear can extend to the spongy body, resulting in the partial or complete rupture of the urethra (3-5).

It is considered a rare urological emergency: an incidence of about 1 in 175,000 has been estimated (2). The incidence, however, is certainly underrated because many patients do not seek medical care for the embarrassment of the condition and/or are often reticent to tell the truth about the cause of the trauma $(6,7)$. Instead, admission to hospital after months from injury is frequent, due to the onset of late complications.

In literature, most cases are reported in the Middle East and North Africa $(8,9)$ where the frequency of the disease is 10 to 100 times higher than in Europe or North America. The incidence of associated urethral injury is instead significantly greater in the United States and Europe, reaching $20 \%$ of cases, compared to Asia, the Middle East and North Africa where it is 3\% $(6,10,11)$. The lesion usually occurs during erection when the albuginea is in tension, and its thickness is reduced to a minimum. The most frequent causes of penile lesion are related to the geographical areas considered. In Western countries the most common cause is sexual intercourse $(6,12-14)$, particularly vigorous, with the penis stroking the bony structures of the pelvis, in 30-50\% of cases (6). In Middle Eastern countries and in North Africa (8, 9, 15-18) fractures are almost always due to handling or masturbation, as a custom widespread in these regions, called "Taqaandan", a maneuver which consists of bending upwards, downwards or sideways, suddenly and vigorously, a part of the penis in erection, keeping the other part in place, to facilitate the detumescence $(8,9)$.

The diagnosis of fractures of the penis is clinical, based on history and physical examination. As a rule, the patient's presentation at the health facilities occurs in the acute phase. The age varies between 12 and 82 years with an average that is concentrated in the fourth decade. 
The symptoms of the penile fracture are various. Patients commonly refer to hearing a sudden clicking sound at the time of the injury, which is comparable to the noise of a bar of glass that breaks $(6,20)$, usually also felt by the partner (21-23). The intensity of the pain, however, is highly variable from patient to patient, and is not directly proportional to the size of the lesion $(20,24)$. It is characterized by quick detumescence accompanied by the appearance of swelling, with or without bruising and deformation of the integument of the shaft. Following the fracture a hematoma is formed; the fascial layers of the genitals determine the distribution and extent of the extravasation: if Buck's fascia remains intact, the extravasation is confined along the penile shaft with typical ovoid "eggplant" distribution; if Buck's fascia is involved, extravasation will expand to the limits of the Colles band resulting in a "butterfly-like" perineal hematoma due to involvement of the scrotum, perineum and the suprapubic area $(8,22,25-29)$. The fracture of the corpora cavernosa may be associated with rupture of the corpus spongiosum of the urethra (approximately 10-20\% of cases) (24). Usually this occurs when there is an extended penile trauma involving both corpora cavernosa.

The history and physical examination of the patient with penile fracture usually makes the use of radiological investigations unnecessary. In any case, the X-ray imaging may be required, especially in patients with atypical clinical presentation (1). The Doppler ultrasound (ECD) is a non-invasive investigation, inexpensive and easy to perform and therefore considered by some authors the radiological investigation of choice in cases of penile trauma. There is also growing evidence of the use of ultrasonography for the evaluation of urethral injury (30). An indirect sign of urethral injury is the presence of air in the corpora cavernosa (31).

The Magnetic Resonance Imaging (MRI) is the most accurate test in diagnosing a fracture of the penis due to its high contrast resolution between tissues and the ability to identify the pathological processes of the soft tissues $(32,33)$. However, it is not widely used because of high costs and long execution times.

Conservative treatment was considered the conduct of choice in case of penile fracture $(22,34)$. However, several studies comparing the long-term results of conservative treatment with the surgical one have demonstrated the superiority of the latter in terms of late complications, in particular the appearance of curvature and erectile dysfunction, better results and shorter hospitalization time $(19,24)$. Therefore, currently immediate surgery is considered the treatment of choice. It allows the evacuation of the hematoma with hemostasis of the blood vessels $(21,35,36)$, the proper cleaning of the injured area and the suture of the albuginea $(22,37)$. The surgery also consists of exploration of the urethra with possible stenting $(37,38)$ or suture of the urethral wound with end-to-end anastomosis (39).

One of the most frequent complications is the penile curvature, secondary to the fibrosis at the site of the trauma; it has been estimated to affect more than $10 \%$ of patients with conservative treatment, percentage reduced to less than a half in patients who undergo surgical treatment (9). The incidence of plaque formation similar to those of La
Peyronie disease (Induratio Penis Plastica), abscesses and penile tissue necrosis reaches up to respectively 25\% and $30 \%$ in patients treated conservatively.

\section{Materials AND MEthods}

In this retrospective study were included all patients with suspected penile fracture that, from January 1987 to June 2013, were admitted to the Urology Clinic at the Hospital of Trieste and the Urology Ambulatory of Associazione Italiana per l'Educazione Demografica (AIED) in Pordenone.

A total of 41 patients were examined, then divided into two groups on a clinical basis (signs and symptoms of acute trauma and post-traumatic long-term complications) and according to the time elapsed between the occurrence of the trauma and the urologic evaluation:

- Group 1 (G1): 23 patients referred from the emergency department with a clinical diagnosis of penile fracture, treated "acutely" or shortly after the trauma (hours-days);

- Group 2 (G2): 18 patients with anamnestic diagnosis of penile fracture, treated at a distance of time from the trauma for the onset of complications (months).

Each patient was evaluated in the following manner:

1. Cause of the trauma;

2. Clinical presentation (signs and symptoms);

3. Radiological imaging (performed or not, type, reliability);

4. Site of the lesion;

5. Urethral involvement;

6. Type of treatment adopted (surgical or conservative);

7. Complications in the short and long term;

8. Re- interventions;

9. Follow-up one year after the first visit;

10.International Index of Erectile Function (IIEF) 15 preand post-trauma;

11.Psychological impact of the trauma on the social and sex life of the patient one year after the trauma, evaluated by psycho-sexological questionnaire created "ad hoc" (satisfaction during sexual intercourse, sexual habits, fear and feelings before and after the trauma, coital pain, interpersonal relationships/anxiety before and after the trauma).

The patients of Gl were subdivided into two subgroups according to the type of treatment adopted (surgical/conservative). For the surgically treated patients, the following data were also taken into consideration:

1. Type of surgery;

2. Incision;

3. Suture (albugineal, Buck's fascia, urethral, dermal);

4. Catheter;

5. Drainage;

6. Dressing and medication.

Most of the data (points 1 to 9) were collected from the analysis of medical records in the general archive and the G2 system of Insiel for patients consulted in Trieste, and from internal archives of patients consulted at the AIED of Pordenone. Since the follow-up data resulted often incomplete, patients were contacted via telephone 
and/or e-mail and invited to the Urology Clinic of the Hospital of Cattinara in Trieste, for an andrological examination. On this occasion it was decided to administer the IIEF 15 questionnaire and also a psycho-sexological questionnaire, which was created specifically after specialist consultation in order to assess the psychological impact of the trauma on the social and sex life of the patient one year after its occurrence. A total of 9 patients of those contacted joined the study.

In G1, the diagnosis of penile fracture was made on a clinical basis, with the exception of two cases with atypical presentation, in which radiological imaging was used, in one case, and surgical exploration, in the other. In more than half of the patients, both to confirm the diagnosis and to evaluate the extent of damage and then decide the most suitable treatment for the situation, radiological examinations were performed (penile ECD in 16 cases; cavernography in 2 cases - one intraoperative; MRI of the lower abdomen in 3 cases, always in association penile ECD). Based on clinical and imaging findings, 3 patients were found to be false positives.

Of the 5 patients with suspected tear in the tunica albuginea with concomitant urethral injury, all patients except one reported hematuria. In the latter case, the radiological imaging result was negative, but the surgery confirmed the diagnosis of urethral involvement.

Uretrography was not performed in any of the cases in order to confirm the diagnosis of urethral injury, unlike what is reported in literature.

In 5 cases, a conservative approach was adopted. Of the remaining patients, 14 underwent surgical exploration with evacuation of the hematoma, suture of the albugineal laceration and eventual closure of the urethral gap, while one patient refused surgery. To patients undergoing conservative treatment medical therapy was set, variable from case to case, consisting mostly of antibiotics, antiinflammatory drugs, and heparin cream for topical use, ice bags, compression bandages, and anti-androgens. In all cases, a one-month long abstinence from sexual intercourse was recommended. 3 of the 5 patients with lesion of the tunica albuginea associated with suspected urethral injury were candidates for repair surgery and the diagnosis was confirmed during surgical exploration.

Patients in G2, with anamnestic trauma and admitted for onset of late complications, have been investigated by medical history and physical examination and subsequently with radiological examinations (dynamic penile ECD in 9 cases; cavernography in 2 cases; ECD + cavernography in 3 cases; MRI of the lower abdomen + ECD in 1 case; $E C D$ + cavernography + MRI in 1 case), which confirmed previous trauma to the corpora cavernosa. Of these 18 patients, 5 were submitted to surgery for the appearance of curvature: in 2 cases corporoplasty for straightening according to the technique of Nesbit was performed; in 1 case the technique of Yachia was employed and in 2 cases patches were used (lyophilized dura patch in one patient, and a venous patch from the right saphenous vein in the other patient).

In order to assess the impact of the fracture on the erectile function and other areas of sexual function, and since the study was retrospective, the patients were asked to fill out two IIEF 15 questionnaires (International Index of
Erectile Function): one in reference to the period before the trauma, the other in reference to the year following the penile fracture.

To complete the assessment of the impact of penile trauma on social life and sex life of the patient, an ad hoc questionnaire was drawn up with the help of a sex therapist, in order to be understandable and completed by the patient himself. With this questionnaire, we tried to investigate the possible difference in satisfaction during sexual relations between pre- and post- trauma, the position taken during fracture in cases of post-coital trauma, possible changes in sexual habits and numerical differences in sexual and/or emotional relationships after the trauma (qualitative analysis with yes/no answers). In addition, the following aspects were evaluated quantitatively by using 7-point Likert scales: fear (of a new trauma, of repercussions on erectile function, of losing sensitivity, of pain during intercourse), penile sensitivity, pain during sexual intercourse, anxiety (in social relations, of performance, of position).

\section{RESULTS}

A total of 41 patients were included in the study, of which 23 belonging to G1 and 18 to G2

In G1 the average age was 40.2 years (range: 26-65) with a prevalence of cases in the third decade (8/23: $34.7 \%$ ); in G2, the average age was 42 years (range: $23-62$ ) with a prevalence of cases in the fifth decade (5/18: 27.8\%). The two groups are therefore age-homogenous. A detailed summary of the characteristics of patients, symptoms, diagnosis and intraoperative findings is described in Table 1.

\section{Diagnosis of penile fracture}

In G1 the diagnosis of penile fracture was suspected in 23 cases, of which 20 (87\%) had a typical clinical presentation (hematoma, swelling, pain, detumescence, noise). In two of these patients (10\%), however, the diagnosis was not confirmed by radiological examinations (negative ECD), which therefore were considered to be false positives (FP). In the 3 cases (13\%) with atypical clinical appearance (pain, detumescence, urethral bleeding), the diagnosis of penile fracture was confirmed in only 2 patients (66.7\%), one case with surgical exploration and the other with radiological imaging, the third resulting therefore FP. Thus excluding from the total count the three FP cases, overall the penile fracture was diagnosed in 20/23 (87\%) patients.

The diagnosis was clinical in 18/20 (90\%) cases and radiological imaging was performed in 14/20 (70\%) (10/14 ECD, 1/14 ECD + cavernography, 3/14 ECD + MRI), which in 13/14 (92.9\%) of the patients confirmed the diagnosis of penile fracture, while 1/14 (7.1\%) cases was negative (negative ECD). In the latter case, the diagnosis of penile fracture was confirmed by surgery.

14 of 20 patients (70\%) underwent surgical exploration; one patient refused the recommended surgery, while 5 of 20 patients (25\%) were treated conservatively. The decision for surgical repair was made on the basis of clinical findings and imaging of the extent of the damage, in cases in which it was performed. 
Table 1. Characteristics, clinical symptoms, intraoperative findings of patients in G1.

\begin{tabular}{|c|c|}
\hline Patients with suspected penile fracture (N) & 23 \\
\hline Average age of patients with range (years) & $40,2(26-65)$ \\
\hline \multicolumn{2}{|l|}{ Causes of trauma } \\
\hline - Sexual intercourse & $18 / 23(\mathbf{7 8 , 6 \% )}$ \\
\hline - Masturbation & $1 / 23(4 \%)$ \\
\hline - Non assessable & $4 / 23(17,4 \%)$ \\
\hline \multicolumn{2}{|l|}{ Clinical signs and symptoms } \\
\hline - Penile hematoma & 19/23 (82,6\%) \\
\hline - Penile swelling & $10 / 23(43,5 \%)$ \\
\hline - Pain & $14 / 23(60,9 \%)$ \\
\hline - Detumescence & $19 / 23(\mathbf{8 2 , 6 \% )}$ \\
\hline - Noise & $8 / 23(34,8 \%)$ \\
\hline - Curvature & $3 / 23(13 \%)$ \\
\hline - Urethral bleeding & $5 / 23(21,7 \%)$ \\
\hline - Erectile dysfunction (ED) & $0 / 23(0 \%)$ \\
\hline Diagnosis of penile fracture & $20 / 23(\mathbf{8 7} \%)$ \\
\hline - Clinical & $18 / 20(90 \%)$ \\
\hline - Clinical (-) with radiological imaging (+) & $1 / 20(5 \%)$ \\
\hline - Clinical (-) with surgical exploration (+) & $1 / 20(5 \%)$ \\
\hline - Radiological imaging & $14 / 20(70 \%)$ \\
\hline $\begin{array}{l}\text { - Radiological confirmation of penile fracture } \\
\text { diagnosis }\end{array}$ & $13 / 14(92,9 \%)$ \\
\hline False positives & $3 / 23(13 \%)$ \\
\hline - Atypical clinical presentation & $1 / 3(33,3 \%)$ \\
\hline - Clinical presentation (+), radiological imaging (-) & $2 / 3(66,7)$ \\
\hline Patients subjected to surgery & $14 / 20(70 \%)$ \\
\hline \multicolumn{2}{|l|}{ Access to the tunica albuginea } \\
\hline - Degloving with circumcision & $11 / 14(78,6 \%)$ \\
\hline - Peno-scrotal skin incision on the median raphe & $1 / 14(7,1 \%)$ \\
\hline - Degloving+second access & $2 / 14(14,3 \%)$ \\
\hline Penile fracture confirmed by surgical exploration & $14 / 14(100 \%)$ \\
\hline \multicolumn{2}{|l|}{ Rupture of the tunica albuginea } \\
\hline - Unilateral & $16 / 20(80 \%)$ \\
\hline - Bilateral & $4 / 20(20 \%)$ \\
\hline \multicolumn{2}{|l|}{ Unilateral rupture of the tunica albuginea } \\
\hline - Right corpus cavernosum & $10 / 16(62,5 \%)$ \\
\hline - Left corpus cavernosum & $6 / 16(37,5 \%)$ \\
\hline Urethral involvement & $5 / 20(25 \%)$ \\
\hline Average of hospitalization days & $4,5(0-11)$ \\
\hline $\begin{array}{l}\text { - Average of hospitalization days of patients } \\
\text { with surgical treatment }\end{array}$ & $5,6(15-3)$ \\
\hline $\begin{array}{l}\text { - Average of hospitalization days of patients } \\
\text { with conservative treatment }\end{array}$ & $2,5(4-0)$ \\
\hline Re-intervention & $3 / 14(21,4 \%)$ \\
\hline
\end{tabular}

Table 2. Long term complications of patients in acute care immediately after the trauma.

\begin{tabular}{|lc|}
\hline . Absence of complications & $12 / 19(\mathbf{6 3 , 1} \%)$ \\
\hline Plaques/nodules along the penile shaft & $7 / 19(36,8 \%)$ \\
\hline Pain/paresthesia & $4 / 19(21 \%)$ \\
\hline$\cdot$ Curvature & $2 / 19(10,5 \%)$ \\
\hline$\cdot$ Urinary disorders & $1 / 19(5,3 \%)$ \\
\hline$\cdot$ Erectile dysfunction (ED) & $2 / 7(28,6 \%)$ \\
\hline
\end{tabular}

In all cases of G2, i.e. 18 patients, the diagnosis was based on remote case history and clinical presentation. We performed a radiological examination in 16 of 18 patients
(88.9\%) (9/16 ECD, 2/16 cavernography, 3/16 ECD + cavernography, 1/16 ECD + MRI, 1/16 ECD + cavernography $+\mathrm{MRI}$ ) confirming the diagnosis of penile fracture in $16 / 16$ (100\%) of the cases. Five of 18 patients (27.8\%) were submitted to surgery and in all cases, corporoplasty was performed for straightening of the penile curvature. Even in this case, the decision for the corrective action was taken based on clinical and imaging findings.

\section{Patients with urethral lesion}

The urethral involvement associated with the laceration of the tunica albuginea was diagnosed in 5/20 (25\%) patients in G1. In 4 (80\%) cases, the cause of the trauma was sexual intercourse, while in 1 (20\%) case it was non assessable. Four of these 5 patients (80\%) reported urethral bleeding; one out of five (20\%) had no suggestive signs or symptoms of urethral injury and even the X-ray imaging (ECD) resulted negative, a fact contradicted by the surgical findings. Of the 16 patients with unilateral rupture of the tunica albuginea, 1 also had a urethral laceration (6.3\%) and 4/4 (100\%) of the patients with bilateral rupture of the albuginea had a concomitant urethral injury. Three of $5(60 \%)$ patients with suspected urethral involvement associated with rupture of the tunica albuginea underwent surgical treatment with simultaneous suturing of the tunica and the urethral mucosa; in 3/3 $(100 \%)$ of the cases the urethral rupture was confirmed by surgical exploration. Among patients treated surgically only one reported post-operative urinary disorders. This patient complained of recurrent urinary tract infections and difficulty in urination; through targeted radiological investigations (voiding cystourethrogram and ECD), he was diagnosed with a urethral stenosis due to the presence of a foreign body granuloma (suture). The patient was re-operated for the removal of the granuloma. Two of 5 patients (30\%) with suspected urethral trauma treated conservatively showed no complications during the follow-up. Of the 18 patients in G2 none had concomitant urethral involvement, as confirmed by the clinical appearance (due to the absence of urethral bleeding in all cases), and by imaging findings.

\section{Follow-up of patients in acute care immediately after the trauma}

Of the 20 patients receiving acute care immediately after the trauma (G1), 14 underwent surgery; no patients (100\%) showed post-surgical complications in the short term. In addition, 9/14 (64.3\%) patients during followup did not show long-term complications, whereas 5/14 (35.7\%) patients had at least one complication. In particular, in 5 cases out of $14(35.7 \%)$ on examination it was possible to appreciate plaques/nodules along the penile shaft, and 3 out of 14 patients (21.4\%) reported pain and paresthesia during sexual intercourse; 1/14 (7.1\%) presented penile curvature, secondary to the corpora cavernosa suturing at surgery, and 1/14 (7.1\%) complained of urinary disorders.

Of the 14 surgically treated patients 3 were re-operated (21.4\%) for the correction of complications: the patient with penile curvature was subjected to corporoplasty, another patient had removal of a foreign body granuloma causing urethral stricture and urinary disorders, 
while the third was subjected to dorsal incision of the prepuce for the onset of phimosis, secondary to ischemia of the skin flaps for excessive devascularization linked to degloving, since he had not been circumcised during the repair surgery of the tunica albuginea.

Among the 5 patients treated conservatively in G1, 3 (60\%) did not present long-term complications. In 2 of 5 patients with late complications (40\%), on physical examination plaques/nodules along the penile shaft were palpable in both cases, and in 1 patient $(20 \%)$ penile curvature was also present. In the latter case, the patient also complained of pain and paresthesia during sexual intercourse. Overall, 19 of 20 patients were available for follow-up, as for one patient with clinical diagnosis of penile fracture, which had refused surgical treatment, it was not possible to collect the documentation. Of these, 12 patients $(63.1 \%)$ did not report any complications, while 7/19 presented plaques/nodules, 4/19 (21\%) pain/paresthesia during sexual intercourse, $2 / 19$ (10.5\%) curvature, and 1/19 (5.3\%) reported urinary disorders. With regard to follow-up about erectile deficiency (ED), 7 of the 20 patients contacted (35\%) agreed to fill out two IIEF 15 questionnaires. The analysis of the pre- trauma IIEF data showed that $7 / 7$ (100\%) patients had no disorder of the sexual sphere, with a total score of 72 to 54 and in all patients the erectile function was between 27 and 30 points. Taking into account the IIEF post-trauma (about 1 year), 2 out of 7 patients (28.6\%) developed post-traumatic ED: mild in one case ( 24 points) and moderately severe in the other (16 points) (Table 2).

\section{Follow-up of patients treated at a distance of time fromthe trauma due to the onset of complications}

Of the 18 patients treated at a distance of time from the trauma (G2) all have manifested long-term complications, as they reported to the urologist just for the onset of symptoms/signs related to the complications. In particular, 8/18 (44.4\%) presented penile plaques/nodules, 4/18 (22.2\%) reported pain and paresthesia during sexual intercourse, $14 / 18(77.8 \%)$ had penile curvature and 1/18 (5.6\%) complained of urinary disorders. In one case of 18 (5.6\%), herniation of the corpora cavernosa was present. In this group of patients, the data related to the ED were collected by consulting the medical records and it was found that 6 patients (33.3\%) experienced an ED (Table 3). Four of the 18 patients contacted responded to the IIEF questionnaire and of these, 3 had an ED. In one case the dysfunction was moderately severe (score of 15) and in two cases mild (score of 19 and 21 respectively).

Table 3. Long term complications in patience treated at a distance of time after the trauma.

\begin{tabular}{|lc|}
\hline$\cdot$ Absence of complications & $0 / 18(0 \%)$ \\
\hline Plaques/nodules along the penile shaft & $8 / 18(\mathbf{4 4 , 4 \% )}$ \\
\hline Pain/paresthesia & $4 / 18(22,2 \%)$ \\
\hline$\cdot$ Curvature & $14 / 18(\mathbf{7 7 , 8} \%)$ \\
\hline$\cdot$ Urinary disorders & $1 / 18(5,6 \%)$ \\
\hline$\cdot$ Hernation of the corpora cavernosa & $1 / 18(5,6 \%)$ \\
\hline$\cdot$ Erectile dysfunction (ED) & $6 / 18(\mathbf{3 3 , 3} \%)$ \\
\hline
\end{tabular}

\section{Follow-up of patients subject to the psycho-sexological questionnaire}

Nine of the 41 patients contacted (22\%) attended the psycho-sexological evaluation. Of these, 7 belonged to G1 and 2 to G2. Four out of 9 (44.4\%) patients interviewed have not noticed any difference in pre- and posttrauma sexual intercourse. The 5 patients (55.6\%) who noticed a difference, responded however in different manners, mentioning: changes in their sexual habits (1 pt.), fear of a new trauma (1 pt.), fear of their partner to cause further trauma (1 pt.), fear of the trauma itself ( $1 \mathrm{pt}$. ), and one patient was not able to answer .

The cause of trauma was in 8 out of 9 cases (88.9\%) sexual intercourse and in 1 case $(11.1 \%)$ related to masturbation. With regard to the position that led to penile fracture, 4 of 8 patients (50\%) reported having had trauma while the woman stood over the man, 3 of $8(37.5 \%)$ while the man penetrated the woman from behind and 1 in $8(12.5 \%)$ while the man stood over the woman. Among patients with post-coital trauma, 4 (50\%) still practice the position that caused the fracture, while 4 (50\%) avoid it: $2 / 4(50 \%)$ because they are afraid of a second trauma, 1/4 (25\%) because their partner is afraid to cause a new fracture and in 1/4 (25\%) because of both coexisting fears. None of the patients surveyed, however, reported a variation in the number of emotional and/or sexual relationships before and after the trauma and none has attributed the cause of the trauma to their personal sex knowledge. Seven out of 9 (77.8\%) patients in the year following fracture reported that they had been afraid of a new trauma, fear that has however diminished with the passage of time. By classifying the level of fear according to a 7-point Likert scale, the level of fear was maximum ( 7 on the Likert scale) in 2 patients, mediumhigh ( 4 and 5) in 3 patients and average low ( 3 and 2 ) in 2 patients. At the time of completing the questionnaire, therefore over a year after the trauma, the level of fear had lowered by about two points in all patients.

Eight of 9 post-trauma patients (88.9\%) were afraid of repercussions on the erectile function, such fear in 2 cases was "very high" ( 7 on the Likert scale), high in 1 case (6), medium-high in 2 cases (4) and medium-low and low in the other 2 cases (2 and 1 ).

The fear of losing penile sensitivity after trauma was reported in 7 out of 9 patients ( $77.8 \%$ ); in these 7 cases the fear was highest in 2 ( 7 on the Likert scale), high in 1 (6), medium-high in another one (5), medium-low in 2 (3 and 2) and low in one other case (1).

Six of 9 patients (66.7\%) had fear of experiencing pain during sexual intercourse after the trauma: in 1 patient the fear was high ( 7 on the Likert scale), in 2 patients medium-high (5 and 4), in 1 medium-low (2) and in 2 cases low (1 on the scale).

The questionnaire also investigated the penile sensitivity pre- and post- trauma, which in 6 of 9 patients $(66.7 \%)$ was found to be unchanged. In the three cases in which a change was reported, for two patients the cause was attributed to surgery, and for one patient to both surgery and trauma itself. Within one year from the trauma, five of 9 patients (55.6\%) experienced pain during sexual intercourse. The intensity of pain varied from patient to patient: in 1 patient it was very high ( 7 on the Likert 
scale), in 1 patient medium-high (4) and in 3 patients very low (1).

The penile fracture in 3 out of 9 patients (33.3\%) increased anxiety in social relations: in 1 patient the anxiety level increased by 2 points on the Likert scale, from 0 (corresponding to "no anxiety") at 2 ("low anxiety"); in 1 patient it augmented from 1 ("very low") to 4 ("moderately high"); in 1 patient the trauma increased anxiety in social relations with a shift from 0 to 7 (corresponding to "very high"). Five of 9 patients (55.6\%) reported an increase in anxiety from performance in response to the trauma: in 1 patient, the increase was from 0 to 1 ; in another patient from 2 to 3 ("moderately low"); in one other case the increase was from 6 ("very high") to 7.

In 2 cases, however, the increased anxiety from performance was more significant, from a score of 0 to 6 in one case and from 0 to 5 in the other case.

The penile trauma also induced in 6 of 9 patients $(66.7 \%)$ an increase in anxiety from position (meaning as "anxiety from position" a fear of experiencing certain positions during intercourse). Of these 6 patients: one patient has gone from 1 to $2 ; 1$ from 0 to $2 ; 1$ other increased from 1 to 7 ; another from 0 to 3 , and finally 2 patients shifted from 0 to 4 .

\section{Comparison between surgically treated patients and conservatively treated patients in Group 1}

The average age of patients treated surgically in Gl was 35.9 years (27-63) and that of the treated conservatively was 43.4 years (26-65). Analyzing the incidence rate of complications developed in the long-term by patients in Gl, it was showed that there is no difference comparing the surgically treated patients $(35.7 \%$, ie $5 / 14$ pcs.) with those treated conservatively $(40 \%$, ie $2 / 5$ pcs.). The data relative to each type of complications are summarized in the table below (Table 4).

In particular, an erectile dysfunction (ED) of mild severity (IIEF of 24) occurred in 25\% (1/4) of the surgically treated patients who responded to the IIEF 15 , unlike patients treated conservatively in which the ED was of modest severity (IIEF of 16 ) in 33.3\% (1/3). The results were compared using Fisher's exact test; from the general analysis and that of each single complication. No statistically significant information was revealed ( $p>0.05$ ) with regard to the correlation between the type of treatment adopted and the onset of complications.

Three out of 14 patients (21.4\%) treated surgically were re-operated for the onset of a complication, while none of the patients to whom medical therapy was prescribed has been subjected to surgery for the onset of complications at a distance of time.

\section{Comparison between patients treated "acutely" and the patients treated at a distance of time for the onset of complications}

The average age of patients in G1 was 40.2 years (26-65) and in G2 of 42 years (23-62): The two groups therefore are fairly homogeneous in respect to age.

The incidence rate of long-term complications was $36.8 \%(7 / 19)$ and $100 \%(18 / 18)$ in G1 and G2 respectively. Erectile dysfunction (ED) of medium/mild severity occurred in $28.6 \%(2 / 7)$ of patients in Gl and of medium/high severity in 33.3\% (6/18) of patients in G2.

The results were compared using Fisher's exact test; from the general analysis of long-term complications in the two groups a $\mathrm{p}$ value $<0.0001$ with a relative risk (RR) equal to 0.3684 were obtained. With these data, it may be concluded that patients treated at a distance of time from the trauma have the probability of manifesting long-term complications 36.8 times higher than those receiving care immediately after the trauma. The specific analysis of each individual complication has not revealed anything statistically significant, except for the occurrence of penile curvature. In this case, in fact, comparing with Fisher's exact test the occurrence rates of this complication in the two different groups, we obtained a $\mathrm{p}$ value $<0.0001$ (RR of 0.1353 and confidence interval (CI) between 0.03564 and 0.5139). Based on these data it can be stated that the treatment at a distance of time from the trauma is likely to lead to penile curvature 13.5 times more than the immediate treatment.

Regarding the ED, according to the results obtained, one cannot assert that patients with treatment at a distance of time have a greater risk of developing ED compared to patients treated immediately after the trauma. The only difference was the degree of ED (mild to moderate in patients of G1/moderate to severe in patients of G2), but other assessments in this matter cannot be made because a few patients were available for completing the IIEF 15 questionnaire and this was the only method used in this study for the evaluation of erectile function. Finally, again from the data obtained, it can be concluded that an inevitable long-term complication in the healing of the rupture of the tunica albuginea, reported in almost all patients, including those undergoing surgical treatment, is the permanent but clinically irrelevant formation of fibrotic scar nodules on the side of the repaired tissue.
Table 4. Comparison of long-term complications.

\begin{tabular}{|lccc|}
\hline Long-term complications & Patients in acute care & Patients treated at a distance of time & P \\
\hline Presence of complications & $7 / 19(\mathbf{3 6 , 8} \%)$ & $18 / 18(\mathbf{1 0 0} \%)$ & $<\mathbf{0 , 0 0 0 1}$ \\
\hline$\cdot$ Plaques/nodules & $7 / 19(36,8 \%)$ & $8 / 18(44,4 \%)$ & n.s. \\
\hline$\cdot$ Pain/paresthesia & $4 / 19(21 \%)$ & $4 / 18(22,2 \%)$ & n.s. \\
\hline$\cdot$ Curvature & $2 / 19(\mathbf{1 0 , 5} \%)$ & $14 / 18(\mathbf{7 7 , 8} \%)$ & $<\mathbf{0 , 0 0 0 1}$ \\
\hline$\cdot$ Herniation & $0 / 19$ & $1 / 18(5,6 \%)$ & n.s. \\
\hline$\cdot$ Urinary disorders & $1 / 19(5,3 \%)$ & $1 / 18(5,6 \%)$ & n.s. \\
\hline$\cdot$ ED & $2 / 7(\mathbf{2 8 , 6} \%)$ & $6 / 18(\mathbf{3 3}, \mathbf{3} \%)$ & n.s. \\
\hline
\end{tabular}

\section{Discussion}

The fracture of the penis is rare urological emergency, even if the total number of cases is certainly underestimated, as many patients do not seek medical care because of embarrassment or report to the urologist belatedly for the occurrence of complications (penile curvature, erectile dysfunction). However, the number of patients 
in our case archive is in line with European case studies. The penile fracture or traumatic rupture of the corpora cavernosa is an injury that occurs in the erect penis. Many Authors $(17,24,25,37)$ in fact recommend that the term "fracture" should be restricted to cases of trauma that occur during penile erection. In Western countries it is mainly caused by sexual intercourse $(6,14)$, in Middle-Eastern countries instead the predominant cause is masturbation $(8,14,40,41)$. The etiological difference in these two regions may be explained by the strict prohibition in the Muslim areas of sexual relations outside of marriage $(6,23)$ resulting in increased frequency of masturbation among the population. The high incidence of penile fracture in these regions of the Mediterranean could be because the time to reach orgasm with penile manipulation is longer than with sexual intercourse, and thus the risk of tearing the tunica albuginea increases. In addition, the preponderance of post-coital penile trauma in Western countries, including the United States, could reflect a more permissive culture in relation to sexual intercourse (6). In this study, the causes of trauma have been sexual intercourse and masturbation in 31/41 (75.6\%) and 2/41 (4.9\%) patients, respectively, reflecting the situation in Western countries. In most cases, the coital trauma occurred while the woman was on top of the man, when the full weight of the partner presses on the erect penis or when the erect penis, out of the vagina, is accidentally pushed against the woman's perineum. In 8 of 41 patients (19.5\%) it was not possible to investigate the etiology of the trauma, and this reflects the natural embarrassment to which this condition can lead $(14,42)$.

In the 23 patients of G1 with suspected penile fracture who reported immediately after the trauma, 19/23 (82.6\%) showed a hematoma along the penile shaft, and in 10 of these $(43.5 \%)$ a swelling of the penis was associated. Pain and detumescence were reported in 14/23 (60.9\%) and 19/23 (82.6\%) cases, respectively. However, only 7 of the 20 patients (35\%) with a confirmed diagnosis of penile fracture confirmed by radiological imaging and/or surgical exploration presented the classical clinical triad (hematoma, noise and immediate detumescence). In literature, noise is reported in 47.7 to $100 \%$ of patients, while pain is present in 48.5 to $100 \%$ of cases, demonstrating a variable constellation of symptoms associated with this condition $(7,9,12,14,34,40,43)$.

In addition to the clinical diagnosis in doubtful cases with atypical clinical appearance, radiological imaging can be made use of to obtain a differential diagnosis of diseases that can mimic penile fracture and thus avoid an unnecessary surgery $(33,34,44)$. In this study the radiological investigations were performed both in clinically typical and atypical cases, for a total of 16 of 23 patients (69.6\%) with suspicion of penile fracture, and this data comes in contrast with information reported in literature. In our case study, we preferred to investigate and ascertain the rupture of the tunica albuginea, drawing on the advice of a great team of radiology specialists with high experience in the field of urology, before subjecting the patient to surgical exploration. All 16 patients who underwent Xray examination were also subject to penile ECD, the radiological technique of choice for evaluating patients with penile trauma $(1,31)$. The anatomy and the penile vasculature can in fact be accurately described by the shades of gray of the Doppler ultrasound technique, so that the nature and extent of the lesion can be fully accounted for in many cases (1). The ECD is able to detect the location of the lesion through the interruption of the echogenic line of the tunica albuginea $(1,31)$. However there have been reports of false-negative results $(1,45,46)$, as pronounced in the presence of a hematoma or edema which render the interpretation difficult (6). In this retrospective analysis, the ECD has confirmed the diagnosis of penile fracture in 13 of 16 cases $(81.3 \%)$, while in 2 cases (12.5\%) it excluded the rupture of the tunica albuginea and in $6.3 \%$ of cases $(1 / 16)$, penile EDC was found to be a false negative. The cavernography is an easy procedure to perform on the operating table, with or without anesthesia (37); however, being a noninvasive diagnostic technique with potential complications such as priapism $(25,47)$, infection (14) and allergic reactions (6), which could lead to cavernous fibrosis $(1,37)$, it was performed in only 2 out of 16 cases (12.5\%): in one case in combination with EDC and intraoperative in the other one. The MRI is a noninvasive procedure, very accurate in the evaluation of penile fracture and that can help determine the most appropriate type of treatment; however because of its high cost (6), it is rarely used in acute situations and many penile trauma cases are been diagnosed only with clinical presentation and ECD. In this study, it has been employed only in $18.8 \%(3 / 16)$ of cases and always in combination with EDC.

The prevalence of urethral lesions in this study was 25\% (5/20) and this is in line with literature, which reports a proportion of cases of urethral involvement between $20 \%$ and $25 \%$ (24). Four out of 5 (80\%) patients with urethral involvement reported urethral bleeding, demonstrating that the lesion of the urethra occurs very commonly with an initial macroscopic presence of red blood cells in the urine. Urethral bleeding (25), hematuria and voiding difficulties $(3,24)$ indeed indicate urethral rupture, however the absence of these signs and symptoms does not exclude possible urethral involvement $(3,5,43,48)$. In medical literature, there have been reports of urethral laceration even in the absence of a rupture of the corpora cavernosa $(13,49)$. The clinical presentation in these cases of isolated urethral injury differs from typical penile fractures for the absence of noise, penile deformity and for the absence of palpable penile defects (49).

As reported in the literature $(6,24)$, in this study the bilateral fracture of the corpora cavernosa is very frequently associated with urethral injury; in fact, 100\% of patients with bilateral rupture of the tunica albuginea had a concomitant urethral injury. In the case of bilateral laceration of the albuginea, the urologist should always give in the operating room special attention to the urethra $(6,14,24)$. Fergany et al. also suggest exploring the other cavernous body when one is broken and the urethra is injured simultaneously.

Three out of 5 (60\%) patients with urethral injury were subjected to surgical repair, but the type of treatment, surgical or conservative, of partial lesions of the urethra remains controversial $(14,42)$. 
In our series of patients with urethral involvement, 1 in $5(20 \%)$ developed a urethral stenosis with associated urinary disorders. Patients with rupture of the tunica albuginea with concomitant urethral injury are much more likely to develop post-operative complications, so a close follow-up is recommended (42).

Some Authors recommend the use of retrograde cystourethrography or urethroscopy in cases of suspected urethral injury $(1,30)$; an indirect sign of urethral injury is the presence of air in the corpus cavernosum $(1,31)$. However, false negative results may be reported $(5,7)$, since the urethrography has limitations and might not detect the tear in the case of post-coital urethral bleeding $(6,23,19)$. None of our cases with suspicion of concomitant urethral involvement, however, has been subjected to such radiological investigations. A direct approach was preferred, looking intraoperative urethral injury (7, 12). Kamdar et al. (7) recommend, in the case of high suspicion of urethral injury, to use a flexible cystoscopy in the operating room prior to inserting the bladder catheter, in order to allow the examination of the urethral mucosa and display the extension of the rupture before placing the catheter.

The urethral injury is much more common when the fracture occurs during sexual intercourse, compared to other causes of penile fracture, and this is because on this occasion the force applied is stronger than during masturbation $(7,8,12,23,24)$. This finding was also confirmed by our study.

In literature it is widely confirmed that immediate surgical repair of the albugineal rupture is the best method of treatment of a penile fracture, leading to excellent results with less time and less risk of hospitalization for erectile dysfunction and curvature in the long term (24). In our case study, 14 of 20 patients (70\%) underwent surgical exploration; of these, 5 patients (35.7\%) had complications during follow-up. Specifically, all 5 patients (35.7\%) presented plaques/nodules along the penile shaft; in addition, 3 of these (21.4\%) reported paresthesia and pain during sexual intercourse, 1 (7.1\%) reported penile curvature and 1 (7.1\%) complained of urinary disorders.

In this study, 1 in 4 (25\%) patients with confirmed diagnosis of penile fracture treated surgically immediately after the trauma, to whom it was possible to administer the IIEF questionnaire, developed post-traumatic ED. This percentage is much higher than the $0-17 \%$ reported in literature $(5,12,14,40)$. This discrepancy may be due to many reasons, first the lack of availability of patients contacted to participate in the study and, consequently, to the scarcity of data obtained from the compilation of the IIEF questionnaire by patients themselves. In addition, the average age of patients treated surgically immediately after the trauma was 35.9 years in our study, an age higher than that reported in several case studies (27-29 years) $(10,34,43)$. An older age may be associated with a higher prevalence of ED before the trauma and consequently this data in our study may have led to a higher percentage of post-traumatic ED. In this context, therefore, the erectile function before the trauma should always be assessed to monitor more precisely any post- traumatic changes, because patients with a preexisting ED are much more prone to deterioration after surgery (14). In our study, erectile function, both pre-and post- trauma, was assessed through the administration of two IIEF questionnaires to the patients. Although the pre- trauma state was estimated retrospectively, and thus represents a source of bias in the study, it is still useful as a reference, although in our series of 4 patients who completed the IIEF, no one had a pre- trauma ED. In any case, this data, albeit limited to the small sample size, cannot be compared in literature, since all of the studies examined lack a pre- evaluation of the erectile function domain of trauma patients who underwent surgical exploration, and there is only sporadic data on this important issue (14). None of our patients with post-traumatic ED, however, necessitated therapy. This suggests that these patients, still able to have satisfactory sexual intercourse, may not seek the attention of the urologist after surgery, even if they suffer from ED, assuming a higher percentage of ED than that reported in literature.

Another important aspect is the adequate evaluation of $\mathrm{ED}$, since in medical literature there is a high variability of measurement criteria $(9,12,41)$, which means that adopting different systems of evaluation leads to equally diverse results and therefore not comparable with each other. The best way to classify ED is the IIEF and penile dynamic ECD (14), but in our study, the penile ECD during follow-up was not performed in all patients. Some patients may show a normal ECD but with a pathological IIEF score in a context of psychological ED (12). In the study of Ibrahiem et al., $36.4 \%$ of men with erectile dysfunction after penile fracture had a normal penile ECD. These patients reported extreme fear of incurring another trauma, and this led to a very limited sex life despite the absence of erectile dysfunction (12). In this regard, the psycho-sexological questionnaire administered to our patients revealed that the only patient with post-traumatic ED in our case series had a very strong fear of impact on the erectile function, of losing penile sensitivity and experiencing pain during sexual intercourse. He also reported, again after the trauma, high social and performance anxiety and a moderate anxiety from position. Taking these data into consideration, therefore, we may consider the ED of this patient is not so much organic, thus linked to the alterations caused by trauma to the erectile tissue and the tunica albuginea, but especially psychological, as described by Ibrahien.

Considering the period of follow-up of patients who have suffered a fracture of the penis, it should long enough, to the point of unmasking any $\operatorname{ED}(40,43)$. In a recent study led by Gamal et al., ED appeared in 4\% of cases after a mean follow-up of 20.8 months (34), while other studies with a longer follow-up of 90 months showed an ED incidence of between 6.6\% and 17\% (5, 12). This relationship is not clear and should be evaluated in future studies (14).

Finally, many reports on penile fracture are based on a patient population of the Middle East $(12,41)$. In the different ethnic groups, however, considering the different etiology of penile fractures, there might be a different percentage of post-traumatic ED, which might explain the higher rate of ED in our study. 
Only one case in 14 (7.1\%) treated surgically developed a penile curvature.

In 13 cases out of 14 (92.8\%), a subcoronal incision with degloving of the penile skin was performed. This surgical technique allows in fact an excellent exposure of both the corpora cavernosa and the corpus spongiosum of the urethra $(6,29)$. In 2 cases, however, a second incision was necessary because the lesion was proximal. In only 1 case a peno- scrotal cutaneous incision was performed on the median raphe, a procedure which avoids excessive disconnection from degloving and delivers good cosmetic results. This incision allows the evacuation of the hematoma and repair of the corpora cavernosa and is recommended in case of widespread edema and hematoma that prevent degloving (7). In addition, it can also serve as a guide for the proximal degloving (50). While the laceration of the tunica albuginea is usually sutured longitudinally along the axis of the penis, the skin closure in the case of peno-scrotal incision could be performed transversely if the longitudinal closure should cause a throttling (51).

The need for catheterization should be evaluated carefully to reduce the risk of infection and possible urethral trauma (15), but in all patients in our study a bladder catheter was positioned to identify the urethra and thus prevent accidental injuries during inspection of the corpora cavernosa to search the albugineal breach, and also to allow possible repair of a concomitant urethral injury. In the absence of urethral laceration in our patients, the catheter was removed in the first post-operative day, while in patients with concomitant urethral injury the catheterization time was longer, in order to allow the urethral suture to stabilize. According to medical literature, in case of simultaneous rupture of the albuginea and the urethra, the catheter may also be left for 7-10 days after surgery (7). The search for the albugineal lesion and therefore the state of the corpora cavernosa and further the sealing of the suture were investigated on the operating table by means of the intracavernous injection of isotonic saline, sometimes mixed with methylene blue dye, causing an artificial tumescence.

This is achieved by placing a tourniquet at the base of the penis. All patients in our case study was finally given a semicompressive dressing after surgery. The post-surgical therapy consisted, in all cases, of abstinence from sexual intercourse for a month, of administration of antibiotics, painkillers/analgesics. Only in one case, an anti-androgen was administered, as normally the postoperative pain prevents erection.

In literature, conservative treatment today is more the exception than the rule, because its results are less satisfactory than those of surgical treatment (6). Conservative therapy is recommended when the corpora cavernosa are intact, but in the case of acute penile fracture, it may be difficult to exclude the complete rupture of the corpora cavernosa, even with a diagnostic radiological procedure $(25,48)$. In our study, five of $20(25 \%)$ patients were treated conservatively. One of these had atypical clinical presentation, but the ECD and MRI were suggestive of penile fracture. Medical therapy adopted involved in the majority of cases the administration of anti-inflammatory/pain relievers, antibiotics, anti-androgens, topical heparin gels, ice bags and the positioning of a compressive dressing which allowed exposure of the glans so as to prevent or detect any penile ischemia $(38,52)$.

Two patients, 1 with clinical diagnosis of penile fracture confirmed by ECD and 1 with only a radiological diagnosis of penile trauma (ECD and positive MRI), have developed long-term complications such as plaques/nodules along the penile shaft in both cases, pain and paresthesia during intercourse and penile curvature in one case (20\%); ED was reported in only 1 patient (20\%).

These data are in contrast with what is described in literature, as the penile curvature and ED following conservative treatment are reported in 50-80\% of cases, for a total of long-term complications after conservative therapy of $80 \%(34,41)$.

In the group treated "acutely" the average number of days of hospitalization (4.5 days overall) was higher in patients who underwent surgical exploration (5.6 days) compared to patients treated conservatively (2.5 days) and this is in stark contrast to the literature that shows the average number of days of hospitalization for patients treated conservatively to be 14 (24). A longer stay in operated patients enrolled in our study may be sought in the presence of cases with concomitant urethral injury, whose post-operative course was linked to the need to maintain the bladder catheter in place for longer, even if the incidence of urethral lesion in our patients was in line with other studies reported in literature. In a study by el-Sherif (15), patients treated conservatively spent on average 2.3 days in the hospital, while those treated surgically 4.1 days. The Author explains this finding by showing that the conservatively treated group had not been catheterized and did not require special medical treatment.

The most common long-term complications of penile trauma are: plaques/nodules along the penile shaft at the site of injury, pain and paresthesia during sexual intercourse, painful erections, penile curvature, erectile dysfunction and urinary disorders. They can occur both in patients treated conservatively and in patients treated surgically (6). In our study, the incidence rate of long-term complications was similar in patients treated surgically immediately after the trauma and in those treated conservatively. In fact, in the first group (treated surgically), the complication rate was $35.7 \%$, while in patients treated conservatively was $40 \%$. This difference in our study is not statistically significant. In light of this data, we can state that the acute conservative treatment does not have a higher risk of long-term complications compared to acute surgical treatment, and that the type of treatment adopted does not affect the overall incidence of long-term complications, which depend on the trauma itself, unlike what is instead reported in literature $(22,34)$.

In this study we also took into consideration 18 patients treated at a distance of time from the trauma for the onset of complications. These patients had not resorted to urological examination immediately after the trauma and therefore had not been subjected to any kind of acute care. They have turned to the doctor in the moment in which long-term complications related to anamnestic penile fracture appeared. One hundred percent of these patients experienced a complication and 
therefore this finding fits with what has been described in literature, as the overall rate of complications after conservative treatment corresponds to $80 \%(34,41)$. In $77.8 \%$ of cases penile curvature was reported, which in $35.7 \%$ of patients was treated surgically because it constituted an obstacle in their intimate life; $33.3 \%$ of cases developed ED. The ratio of penile curvature in this group of patients was in line with the data for post-conservative treatment curvature reported in the literature (50-80\%) $(34,41)$, while the incidence of ED was lower $(50-80 \%)$. This can be explained by the fact that many patients with previous penile trauma, who had a mild ED that did not prevent normal sexual relations, have not felt the need for a urological examination.

Comparing the incidence rates of long-term complications of patients receiving acute care (G1) and patients treated at a distance of time (G2), it was shown that nonimmediate treatment after the trauma has a $36.8 \%$ risk of leading to complications and in particular a $13.5 \%$ risk of generating a penile curvature. From these data it may be concluded that the acute treatment of a penile fracture would be more suitable, reducing the overall risk of long-term complications secondary to trauma, particularly the risk of penile curvature. The immediate treatment, whether surgical or conservative, then turns out to be the ideal treatment of penile fractures, but it the choice does not depend on the doctor, but rather the patient, which for the embarrassment generated by the situation does not seek medical care immediately and requires specialist advice only when complications are already notable. Given the small number of patients who responded to the IIEF, we do not have sufficient data to enable us to assess the post-traumatic ED. However, the lack of participation in completing the IIEF questionnaire may presume a certain well-being of patients, who then have no interest in contacting the urologist.

From the analysis of the psycho-sexological questionnaire no conclusions can be drawn, as only few patients from an already small group, were available to answer the questions submitted. This might also indicate a reluctance to face again the traumatic event, perhaps to relive the negative experience, or, on the contrary, it could indicate a certain well-being of the patient, who does not feel the need to undergo a medical evaluation. Considering all the above, the interpretation of the data can be very variable.

This questionnaire, not being validated, but purposebuilt by a sex therapist to investigate certain personal aspects not investigated by the IIEF, does not allow for a quantitative analysis of data; it only allows a qualitative analysis of data that differ greatly from patient to patient. However, we can affirm that the penile fracture definitely has a psychological impact on the patient, especially in the year following the trauma. The psychological sphere that is mainly affected is that of fear. The majority of patients admitted in fact that in the year following the trauma they experienced fear of new trauma, fear that in several cases was experienced also by the partners. The same thing can be said about the fear of the repercussions on erectile function, fear of losing penile sensitivity and fear of experiencing pain during intercourse. However, all these fears diminish with the passage of time, perhaps for the reacquisition by the patient of selfconfidence, and having ascertained that despite the trauma "everything was back to normal".

Despite the fear, patients have not noticed differences in sexual relationships before and after the trauma; the few patients who have noticed these differences, however, gave very different answers, so it was difficult to make a quantitative analysis of the data.

In general, even anxiety in social relationships, anxiety from position and performance have not undergone much change between the pre- and post- trauma states.

\section{Limitations of the study}

This study is limited by the number of patients, especially in comparison with other studies from the Middle East or the Mediterranean basin $(9,12,41)$. In our case series, already numerically limited from the start, it was possible to evaluate the erectile function and psycho- sexological impact of the trauma on the emotional and sex lives of the patients only in 9 cases out of a total of 41 ; therefore, the interpretation of the data obtained has proven difficult in some respects. Many patients with penile fracture have not turned to the doctor for their possible welfare and/or the non-occurrence of complications. However, this is not clear and must be taken into account when interpreting the results of this study. In addition, the retrospective evaluation of patient status before the injury might have caused some bias, but this should be considered as a benefit of the study, since the evaluation before the occurrence of a penile fracture is not possible.

\section{Conclusions}

The fracture of the penis is a rare urological emergency, even if underestimated, probably because of socio-cultural inhibition of the patients, who are ashamed to report to the doctor. Sexual intercourse and penile manipulation are the most frequent causes of this type of trauma, depending on the geographical area analyzed. The radiological imaging does not replace clinical evaluation, but in our experience, it is very useful in cases with atypical clinical presentation and in cases of doubt, especially because it allows assessing the need for surgical exploration. The penile Doppler ultrasound is the radiological technique of choice, given its diagnostic accuracy, non-invasiveness, speed of execution and low cost.

A concomitant urethral injury must always be taken into account especially in case of urethral bleeding or bilateral lesion of the corpora cavernosa.

In the literature, the surgical technique most recommended is the incision with sub-glandular de-gloving of the penile skin, which allows a good exposure and viewing of the corpora cavernosa and the corpus spongiosum of the urethra, in order to simultaneously repair the tunica albuginea and the eventual urethral injury.

In our study, in contrast to what is reported in literature, we found no difference in the onset of long-term complications among patients treated surgically compared to those treated conservatively. Instead, comparing patients treated "acutely" with those treated at a distance of time after the trauma, it was found that the latter have a higher probability of experiencing complications, and in par- 
ticular of experiencing penile curvature. However, taking into account the small number of patients who were available for filling the IIEF 15 questionnaire, we cannot state that patients treated after a long time have a higher risk of developing ED than those treated immediately after the trauma. From the results obtained, we can only say that in the two groups ED occurred with varying severity: moderate-to-severe in those treated non-immediately, medium-low in the "acutely" treated.

In conclusion, the immediate treatment of a penile fracture (both surgical and conservative) certainly offers a greater chance of healing without complications, preserving the erectile function and preventing the onset of pain and penile curvature, compared with no treatment. The analysis shows that the psicosexological trauma definitely has an impact on social life and sex life of the patient.

Anxieties from position and performance remain unchanged, while fear, in its various aspects, is the emotion most frequently detected; however, it decreased over time, and this is also confirmed by the fact that in most cases follow up was interrupted one year after the trauma due to general well-being of the patients.

\section{References}

1. Bertolotto M, Calderan L, Cova MA. Imaging of penile traumastherapeutic implications. Eur Radiol. 2005; 15:2475-82.

2. Koifman L, Barros R, Junior RA, et al. Urology. 2010; 76:1488-92.

3. Tsang T, Demby AM. Penile fracture with urethral injury. J Urol. 1992; 147:466-8.

4. Safarinejad MR, Lashkari MH, Babaei A, et al. Penile vascular indices in surgically treated and conservatively treated penile fracture: does conventional immediate repair matter? Int Urol Nephrol. 2012; 44:1631-40

5. El-Assmy A, El-Tholoth HS, Mohsen T, Ibrahiem el HI. Long-term outcome of surgical treatment of penile fracture complicated by urethral rupture. J Sex Med. 2010; 7:3784-8.

6. Eke N. Fracture of the penis. Br J Surg. 2002; 89:555-5.

7. Kamdar C, Mooppan UM, Kim H, Gulmi FA. Penile fracture: preoperative evaluation and surgical technique for optimal patient outcome. BJU Int. 2008; 102:1640-4.

8. Zargooshi J. Penile fracture in Kermanshah, Iran: report of 172 cases. J Urol. 2000; 164:364-366.

9. El Atat R, Sfaxi M, Benslama MR, et al. J Trauma 2008; 64:121-5.

10. Zargooshi J. Penile fracture in Kermanshah, Iran: the long-term results of surgical treatment. BJU Int. 2002; 89:890-4.

11. Derouiche A, Belhaj K, Hentati H, et al. Management of penile fractures complicated by urethral rupture. Int J Impot Res. 2008; 20:111-4.

12. Ibrahien el-HI, el-Tholoth HS, Mohsen T, et al. Penile fracture: long-term outcome of immediate surgical intervention. Urology. 2010; 75:108-11.

13. Cortellini P, Ferretti S, Larosa M, et al. Traumatic injury of the penis: surgical management. Scand J Urol Nephrol. 1996; 30:517-9.

14. Hatzichristodoulou G, Dorstewitz A, Gschwend JE, et al. Surgical management of penile fracture and long-term outcome on erectile function and voiding. J Sex Med. 2013; 10:1424-30.
15. el-Sherif AE, Dauleh M, Allowneh N, Vijayan P. Management of fracture of the penis in Qatar. Br J Urol. 1991; 68:622-5.

16. Taha SA, Sharayah A, Kamal BA, et al. Fracture of the penis: surgical management. Int Surg. 1988; 73:63-4.

17. Mansi MK, Emran M, el-Mahrouky A, el-Mateet MS. Experience with penile fractures in Egypt: long-terms results of immediate surgical repair. J Trauma. 1993; 35:67-70.

18. Al Saleh BM, Ansari ER, Al Ali IH, et al. Fractures of the penis seen in Abu Dhabi. J Urol. 1985; 134:274-5.

19. Nicolaisen GS, Melamud A, Williams RD, McAninch JW. Rupture of the corpus cavernosum: surgical management. J Urol. 1983; 130:917-9.

20. Jack GS, Garraway I, Reznichek R, Rajfer J. Current treatment options for penile fractures. Rev Urol. 2004; 6:114-20.

21. Cendron M, Withmore KE, Carpiniello V, et al. Traumatic rupture of the corpus cavernosum: evaluation and management. J Urol. 1990; 144:987-91.

22. Muentener M, Suter S, Hauri D, Sulser T. Long-term experience with surgical and conservative treatment of penile fracture. J Urol. 2004; 172:576-9.

23. Asgari MA, Hosseini SY, Safarinejad MR, et al. Penile fractures: evaluation, therapeutic approaches and long-term results. J of Urol. 1996; 155:148-149.

24. Fergany AF, Angermeier KW, Montague DK. Review of Cleveland Clinic experience with penile fracture. Urology. 1999; 54:352-5.

25. Hinev A. Fracture of the penis: treatment and complications. Acta Med Okayama. 2000; 54:211-16.

26. Garaffa G, Raheem AA, Ralph DJ. Penile fracture and penile reconstruction. Curr Urol Rep. 2011; 12:427-31.

27. De Giorgi G, Luciani LG, Valotto C, et al. Early surgical repair of penile fractures: our experience. Arch Ital Urol Androl. 2005; 77:103-5.

28. Gontero P, Sidhu PS, Muir GH. Penile fracture repair: assessment of early results and complications using color Doppler ultrasound. Int J Impot Res. 2000; 12:125-8.

29. Miller S, Mc Aninch JW. Penile fracture and soft tissue injury. In: Mc Aninch JW, ed. Traumatic and Reconstructive Urology. Philadelphia: W.B. Saunders; 1996; 693-698.

30. Pavlica P, Barozzi L, Menchi I. Imaging of male urethra. Eur Radiol. 2003; 13:1583-96.

31. Bertolotto M, Mucelli RP. Nonpenetrating penile traumas: sonographic and Doppler features. AJR Am J Roentgenol. 2004; 183:1085-9.

32. Choi MH, Kim B, Ryu JA, et al. MR imaging of acute penile fracture. Radiographics. 2000; 20:1397-405.

33. Murray KS, Gilbert M, Ricci LR, et al. Penile fracture and magnetic resonance imaging. Int Braz J Urol. 2012; 38:287-8.

34. Gamal WM, Osman MM, Hammady A, et al. J Trauma 2011; 71:491-3.

35. Nehru-Babu M, Hendry D, Ai-Saffar N. Rupture of the dorsal vein mimicking fracture of the penis. BJU Int. 1999; 84:179-80.

36. Sant GR. Rupture of the corpus cavernosum of the penis. Arch Surg. 1981; 116:1176-8.

37. Klein FA, Smith MJ, Miller N. Penile fracture: diagnosis and management. J Trauma. 1985; 25:1090-2. 
38. Kowalczyk J, Athens A, Grimaldi A. Penile fracture: an unusual presentation with lacerations of bilateral corpora cavernosa and partial distruption of the urethra. Urology. 1994; 44:599-601.

39. De Rose AF, Giglio M, Carmignani G. Traumatic rupture of the corpora cavernosa: new physiopathologic acquisitions. Urology. 2001; 57:319-22.

40. Ateyah A, Mostafa T, Nasser TA, et al. Penile fracture: surgical repair and late effects on erectile function. J Sex Med. 2008; 5:1496502.

41. Zargooshi J. Sexual function and tunica albuginea wound healing following penile fracture: An 18-year follow-up study of 352 patients from Kermanshah, Iran. J Sex Med. 2009; 6:1141-50.

42. Sawh SL, O'Leary MP, Ferreira MD, et al. Fractured penis: a review. Int J Impot Res. 2008; 20:366-9.

43. Mydlo JH. Surgeon experience with penile fracture. J Urol 2001; 166:526-8

44. Kachewar S, Kulkarni D. Ultrasound evaluation of penile fractures. Biomed Imaging Interv J. 2011; 7:e27.

45. Martinez Perez E, Arnaiz Esteban F, Perez Arbej JA, et al.
Fractura de pene: dos nuevos casos. Revision de literatura. Utilidad de la ecografia. Arch Esp Urol. 1997; 50:1099-1102.

46. Koga S, Saito Y, Arakaki Y, et al. Sonography in fracture of the penis. Br J Urol. 1993; 72:228-9.

47. De Stefani S, Stubinski R, Ferneti F, et al. Penile fracture and associated urethral injury. Scientific World Journal. 2004; 4 Suppl 1:92-9.

48. Mydlo JH, Hayyeri M, Macchia RJ. Urethrography imaging in a small series of penile fractures: a comparison with surgical findings. Urology. 1998; 51:616-19.

49. Mohapatra TP, Kumar S. Reverse coitus: mechanism of urethral injury in male partner. J Urol. 1990; 144:1467-8.

50. Su LM, Sutaria PM, Eid JF. Repair of penile rupture through a high-scrotal midline raphe incision. Urology. 1998; 52:717-9.

51. Karadeniz T, Topsakal M, Airman A, et al. Penile fracture: differential diagnosis, management and outcome. Br J Urol. 1996; 77:279-81.

52. Orvis BR, McAninch JW. Penile rupture. Urol Clin North Am. 1989; 16:369-75.

\section{Correspondence}

Nicola Pavan, MD (Corresponding Author)

nicpavan@gmail.com

Giorgia Tezzot, MD

giorgiatez@hotmail.it

Giovanni Liguori, MD

gioliguori@libero.it

Renata Napoli, MD

renata.napoli@libero.it

Paolo Umari, MD

paoloumari@alice.it;

Michele Rizzo, MD

mik.rizzo@gmail.com

Giovanni Chiriacò, MD

gio.chiriaco@gmail.com

Gaetano Chiapparrone, MD

g.chiapparrone@gmail.com

Francesca Vedovo, MD

superv@libero.it

Carlo Trombetta, MD

trombcar@units.it

Urology Department, Cattinara Hospital, University of Trieste

Strada di Fiume 447 - 34149 Trieste, Italy

Michele Bertolotto, MD

bertolot@units.it

Radiology Department, Cattinara Hospital, University of Trieste

Strada di Fiume 447 - 34149 Trieste, Italy 